\title{
COMPUTATIONAL INTELLIGENCE APPLIED TO WILDFIRE PREDICTION USING WIRELESS SENSOR NETWORKS
}

\author{
V. G. Gasull ${ }^{1}$, D. F. Larios ${ }^{1}$, J. Barbancho ${ }^{1}$, C. León ${ }^{1}$ and M. S. Obaidat ${ }^{2}$ \\ ${ }^{I}$ Department of Electronic Technology, University of Seville, Seville, Spain \\ ${ }^{2}$ Department of Computer Science \& Software Engineering, Monmouth University, W. Long Branch, NJ 07764, U.S.A. \\ \{vgasull,dflarios\}@dte.us.es, \{jbarbancho,cleon\}@us.es,obaidat@monmouth.edu
}

\begin{abstract}
Keywords: $\quad$ Fuzzy system, Wireless sensor networks, Forest fire, Simulation.
Abstract: In this paper we present a novel algorithm, implemented in a wireless sensor network environment, for the study of fire in a natural environment. This algorithm can offer two kinds of information: the risk of a fire and, in case of an active fire, the propagation direction of the flames and their velocity. This information is useful for fire rangers in order to obtain escape ways and determine strategies for putting off the fire. The evaluation of the proposal algorithm is performed using an ad-hoc simulator. The preliminary results obtained show that the proposed algorithm is a promising method that can be used to solve the most common problems related to fire control and detection.
\end{abstract}

\section{INTRODUCTION}

Wireless sensor networks (WSNs) has been applied to a wide range of applications, such as agriculture, military, medical, industrial, environmental and habitat monitoring, among others (Akyildiz et al., 2002). One of the possible implementations of WSN in natural environments is the study of forest fires; based on preventing, detecting or predicting the behavior of fire in a forest fire. Usually, a wireless sensor network is composed of multiple nodes, distributed on an area, which used data aggregation to obtain information about the environment through the signals gathered from the sensors.

Several research works that implement a WSN for forest fire applications are presented in the literature. Most of them base their research on the detection of fire, such as (Kosuku et al., 2009) which uses a WSN to gather information to be used in the FARSITE simulator. In (Ramachandran et al., 2008) a WSN based on a swarm-inspired system is used for detecting wildfires. It is a probabilistic model that focuses on responses for query requests in an accurate manner. In (Zhang et al., 2009) a WSN with a satellite monitoring system is used for forest fire detection. Reference (Li et al., 2009) presents an implementation scheme of communication oriented WSN and monitoring computer using VB6.0 and SQL SERVER 2000. The work in reference
(Hefeeda and Bagheri, 2007) is meant to reduce the consumption on transmitting the information using data aggregation by analyzing the Fire Weather Index (FWI) System. The work reported in (Yu et al., 2005) uses a neural network system to prolong the lifetime of the sensor.

Some other works are related to the study of fire evolution (Lei and $\mathrm{Lu}, 2010$ ), fire prevention (Obregon et al., 2009). Others realize real experiments with WSN in order to evaluate its robustness against real conditions of a wildfire (Antoine-Santoni et al., 2009).

Other works are related to the use of WSN to improve the security on evacuations (Cherniak and Zadorozhny, 2010). Reference (García et al., 2008) deals with the use of WSN to improve the information gathering for fire-fighters, in order to allow a better actuation to extinguish the fire.

Usually, we use a system that is based on a WSN to collect the sensor information and a centralized PC to process the information to obtain the various parameters of fire forests to assess, for example the risk of fire. Only a few distributed approach are proposed, like the one in (Liu et al., 2009), where the remote nodes process information of multiple sensors (temperature and smoke) sending alarm to a base station if a node detect an incident.

However, centralized algorithms could be a problem in real situations since if some nodes fail because they are burned, a path to the base station 
could disappear. Our proposal is based on a distributed processing, that can transmit the information to a nearby operator. Our scheme does not need a base station, and offers two types of information: local information and global information. A fire fighter can access this information with a portable device on every node of the network.

For a fire to take place, it is necessary to have the coincidence in time and space of different factors. These factors are summed-up in the Figure 1; it is called the triangle of Fire. In a typical wildfire, fuel is wood and vegetation and the oxidizing are the air. Apart from fuel and oxidization, it is necessary to have activation energy. In a wildfire, the energy can be originated by various factor, like lightning, replica, negligence, deliberated and unknown causes, but in most of cases (around a 95\%), it is caused by human.

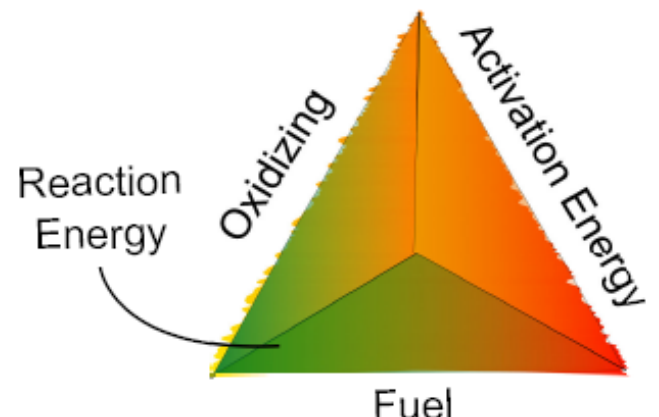

Figure 1: Triangle of fire.

Because of that, in order to evaluate the risk of fire it is necessary to consider sociological information, not only environmental condition. The proposed algorithm, called ISFPWSN (Intelligent System for Fire Prediction using Wireless Sensor Networks), takes this information into account in order to evaluate the risk of fire.

Apart from the risk of fire, other goal of ISFPWSN is to offer information about the behavior and evolution of the fire in case of wildfire. It reduces the risk of the people exposed and improves the fire detection because it offers information about secure ways of escape and permits evaluation of a strategy for fire extinguishing.

ISFPWSN is based on computational intelligence algorithms that use fuzzy inference systems. It is because fuzzy system has many advantages for WSN applications (Marin-Perianu and Havinga, 2007), such as its simplicity, which permits execution on devices with limited capabilities, or its ability to manage imprecise and uncertain information. All of these characteristics allow us to obtain a robust system without a high computational load.

The remainder of this paper is organized as follow: Section 2 presents the proposed system ISFPWSN. On Section 3 the simulator developed for test ISFPWSN is described. Finally, in Section 4 we present concluding remarks and provide a discussion of future works.

\section{PROPOSED SYSTEM}

ISFPWSN is designed using a wireless sensor network, as it described on section 2.3. The operation of the system can described as below:

The nodes of the network execute their local processing algorithms to obtain the local estimates. Then they send these local estimates other nodes in the network. Every node of the network gathers its information and executes the distributed processing algorithms. Subsequently, all the nodes share their information about the risk of fire or, in case of a forest fire, its evolution. ISFPWSN does not need a base station to gather the information. This increases the robustness of the network in case of wildfire, because if some nodes were damaged from the fire, it is possible that they do not exit a route to send the information to the base station.

On the other hand, since all the nodes share the estimation and behavior of the fire, a firefighter can access to this information anywhere on the network, which is a great aspect. ISFPWSN is based on two processes: a local process and a distributed process, these processes are described in sections below.

\subsection{Local Processing}

Local processing is executed on every node of the network. Nodes evaluate the state of the fire with the information provided by the sensors connected up to the nodes. This algorithm offers a partial solution of the global state of the system since this method only uses local information to get the results. The proposed local processing is basically a fusion and data aggregation.

Within local processing there are two algorithms; an algorithm to obtain the probability of fire occurrence (PF) and an algorithm to obtain the behavior of fire $(\mathrm{BF})$.

\subsubsection{Probability of Fire Occurrence (PF)}

This algorithm determines the risk of fire in the environment in the neighborhood of the node. The 
summary of algorithm is shown Figure 2.

The output of the PI algorithm is a value between 0 and 100, which indicates the probability of ignition of a forest fire after normalization. This algorithm is divided up in two blocks: an environmental processing and a sociological processing. Both methods are based on a fuzzy logic engine.

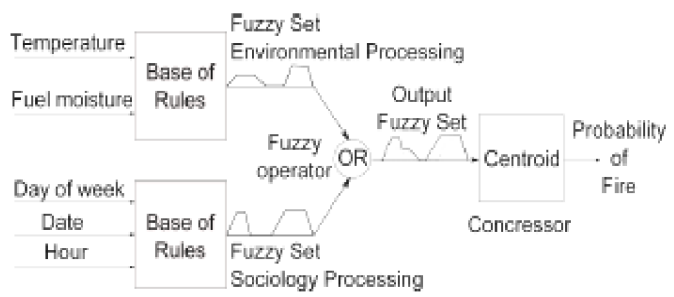

Figure 2: Algorithm of the probability of fire.

Table 1 summarizes the fuzzy sets of the output on both processing outputs (environmental and sociological).

Table 1: Fuzzy sets of the outputs of environmental and sociological processing.

\begin{tabular}{|c|c|}
\hline Name & Membership function \\
\hline Very low & Trapezoid $(0,0,10,17)$ \\
\hline Low & Triangle $(13,25,37)$ \\
\hline Median & Triangle $(35,47,59)$ \\
\hline High & Triangle $(57,69,81)$ \\
\hline Very high & Trapezoid $(79,90,100,100)$ \\
\hline
\end{tabular}

- Environmental Processing: This processing method tries to obtain the fire likelihood as a function of the environmental variables. This is the classical assumption considered in others fire simulators. Most simulators base his calculations on the Rothermel model (Rothermel, 1983) or the FWI index (Canadian Forest Fire Danger Rating System, 1985). The input variables considered for this process are temperature and fuel moisture. Because of historical data of fires, these magnitudes appear as the most relevant ones. The fuzzy sets of the inputs are shown in table 2.

The environmental processing has the base of knowledge described on Table 3.

- Sociological Processing: This processing is intended to obtain the fire likelihood as a function of the sociological variables. Figure 3 shows the causes of forest fires in Spain during the period 1996-2005. The figure shows that approximately $95 \%$ of the wildfires are caused by the human. Moreover, a great percentage of these wildfires are produced in weekend. Because of it, we think it is necessary to consider sociological variables to estimate the risk of fire. Despite its importance, this kind of information, in general, is not considered by fire simulator because it if difficult to assess. This is because sociological behavior is uncertainly and it is not easy to model with classic techniques. Fuzzy logic is a good approach to evaluate this kind of behavior.

Table 2: Fuzzy sets of the inputs of the environmental processing.

\begin{tabular}{|c|c|c|}
\hline Name & Set & Membership function \\
\hline \multirow{4}{*}{ Temperature } & Low & Trapezoid $(-10,-10,20,40)$ \\
& Median & Triangle $(15,30,45)$ \\
& High & Trapezoid $(35,65,100,100)$ \\
\hline \multirow{2}{*}{ Fuel } & Low & Trapezoid $(0,0,10,30)$ \\
Moisture & Median & Triangle $(20,40,60)$ \\
& High & Trapezoid $(35,65,100,100)$ \\
\hline
\end{tabular}

Table 3: Base of knowledge of the environmental processing.

\begin{tabular}{|c|c|c|}
\hline Temperature & F. Moisture & Output \\
\hline Low & High & Very low \\
\hline Low & Median & Low \\
\hline Low & Low & Medium \\
\hline Median & High & Low \\
\hline Median & Median & Medium \\
\hline Median & Low & High \\
\hline High & High & Medium \\
\hline High & Median & High \\
\hline High & Low & Very High \\
\hline
\end{tabular}

By analyzing this information, we can deduce that in most cases the risk of fire. Most importantly the number of visitor to the natural park is considered more important than the environmental variables. Because of that, is important to consider information such as holidays or weekends to estimate the probability of fire. Considering this information is the mission of the sociological processing. This system is also implemented with a fuzzy logic engine. The input variables are day of week, date and time/hour. The considered fuzzy sets are summarized in Table 4. A low season for park visits is considered between November and April while the high season considered between May and October. Low visit hours represent times when it is cold during the day while high visit hours are the hours when it is hot during the day. 


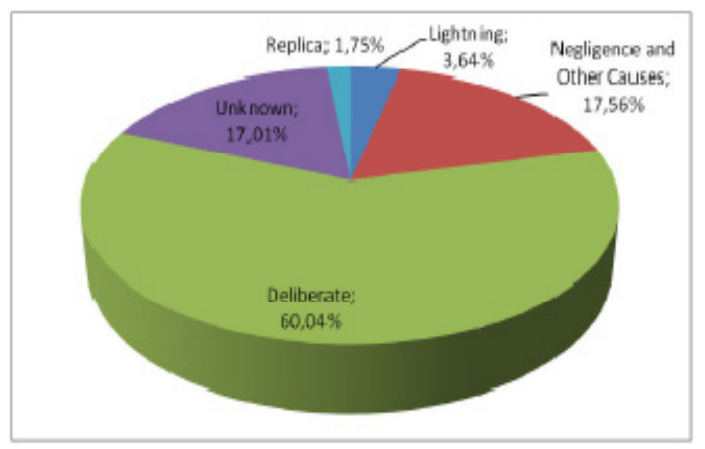

Figure 3: Causes of Wildfires.

Table 4: Fuzzy sets of the inputs of the sociological processing.

\begin{tabular}{|c|c|c|}
\hline Name & Set & Membership function \\
\hline \multirow{2}{*}{ DOW } & $\begin{array}{c}\text { Mon-Thurs } \\
\text { Fri-Sun }\end{array}$ & $\begin{array}{c}\text { Trapezoid }(0,0,3,3) \\
\text { Triangle }(3,5,7)\end{array}$ \\
\hline \multirow{2}{*}{ Date } & Low & Trapezoid $(0,2,5,8)$ \\
& High & Triangle $(6,9,12)$ \\
\hline \multirow{2}{*}{ Hours } & Low & Trapezoid $(0,3,11,14)$ \\
& High & Triangle $(12,17,24)$ \\
\hline
\end{tabular}

It is important mention that these rules apply to Spain, where the dry months are between April and November. The Sociological processing has the base of knowledge described in Table 5.

\subsubsection{Fire Behavior (FB)}

This algorithm obtains the direction of the fire front and its velocity in an area around the node. It does not consider the topology of the terrain; it is used on the distributed algorithm for prediction of direction and velocity of the fire.

This algorithm considers the nodes as isolated devices with their local information of wind direction and velocity, temperature, humidity and daily rain. This algorithm is executed only in case of fire.

The velocity of propagation of the fire is calculated using the formula below:

$$
\mathrm{V}=\mathrm{a}(\mathrm{U}+1) \mathrm{b}
$$

where $\mathrm{V}$ is the velocity of propagation of fire $(\mathrm{m} / \mathrm{min}), \mathrm{U}$ is the wind velocity $(\mathrm{m} / \mathrm{s}), \mathrm{a}=0.233$ and $\mathrm{b}=1.332$ for a "Pinnus Pinea" forest, as we consider in this work. The direction of propagation can be obtained as follows:

- If wind velocity $=0 \mathrm{Km} / \mathrm{h}$ : The fire follows a radial pattern, with the same velocity on all direction
- For other cases: The fire front has the direction of the wind and will trace an ellipse

Table 5: Base of knowledge of the sociological processing.

\begin{tabular}{|c|c|c|c|}
\hline Day of week & Date & Hour & Output \\
\hline Monday-Thursday & Low & Low & Very low \\
\hline Monday-Thursday & Low & High & Low \\
\hline Monday-Thursday & High & Low & Low \\
\hline Monday-Thursday & High & High & High \\
\hline Friday-Sunday & Low & Low & Low \\
\hline Friday-Sunday & Low & High & Median \\
\hline Friday-Sunday & High & Low & Median \\
\hline Friday-Sunday & High & High & Very high \\
\hline
\end{tabular}

This algorithm also implements an alarm system for risk of major fires, according to the rule of $30 \%$. This rule says that a great forest fire can be produced with the conditions described on Table 6.

Table 6: Rule of $30 \%$.

\begin{tabular}{|c|c|}
\hline Parameter & Value \\
\hline Temperature & $>30 \%$ \\
\hline Humidity & $<30 \%$ \\
\hline Wind velocity & $>30 \mathrm{~km} / \mathrm{h}$ \\
\hline Days without rain & $>30$ \\
\hline
\end{tabular}

\subsection{Distributed Processing}

The distributed processing is executed on every node of the network; with the information gathered from the broadcast messages sent by all nodes in the network. This permits checking the global state of the environment without the need for a base station that collect all the information. This is especially useful for the study of wildfire as the route between any nodes to the base station can disappear if any amounts of nodes are burned by the wildfire. Moreover, this allows obtaining the global information, anywhere into the network. It can help fire-fighters who only need a small mobile device to check the evolution of the wildfire.

The distributed processing scheme combines all the partial solutions from the local processing of the nodes with two other algorithms: risk of fire and prediction of the direction and the velocity of the fire algorithms. These algorithms provide results to assess the overall state of the environment.

\subsubsection{Risk of Fire (RF)}

This algorithm evaluates the global risk of fire in the 
environment. In this case, it combines the results of every node in the network using the next formula:

$$
R F=\frac{\sum \mathrm{PF}_{\text {nodes }}}{\mathrm{N}}
$$

Where $\mathrm{N}$ is the number of nodes, PFnodes is the global estimation of the probability of fire and RF represents the risk of fire.

The output of this algorithm is a value between 0 and 100, which will be encoded in one of five possible risk levels that have been considered. Values above 60 represent a high risk of forest fire. Its output value can be classified according to their value as described on Table 7.

\subsubsection{Prediction of Direction and Velocity of the Fire (PD)}

This algorithm determines the global characteristic of the wildfire. This information is useful for the firefighters in order to determine the best way to extinguish a fire and the route for escape to be used which increases the safety aspect of the approach.

Table 7: Evaluation of the risk of fire.

\begin{tabular}{|c|c|}
\hline Value RF & Probability of Fire \\
\hline $\mathrm{RF} \leq 10$ & Very Low \\
\hline $10<\mathrm{RF} \leq 30$ & Low \\
\hline $30<\mathrm{RF} \leq 40$ & Median \\
\hline $40<\mathrm{RF} \leq 60$ & High \\
\hline $\mathrm{RF}>60$ & Very High \\
\hline
\end{tabular}

This algorithm is based on a fuzz logic system and offers two classes of outputs: the directions of the fire and its velocity.

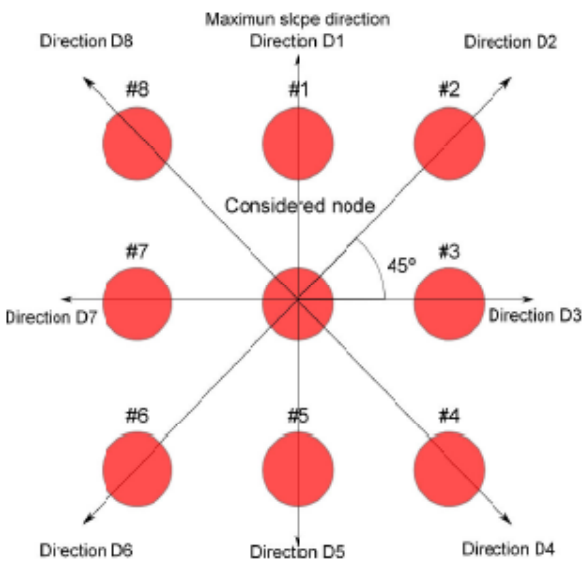

Figure 4: Considered directions of the fire.

Direction of fire is divided into 8 direction (D1 to D8), with 45 degrees between them and with D1 representing the direction of the node with the maximum slope in relation with a considered node. This is represented in Figure 4. Every Di variable can only have two values: on or off. On signifies that the fire is going to advance in its direction. Off signifies that fire is not going to advance in it direction.

The velocity of propagation is represented by five values: very low, low, medium, high and very high.

As inputs, this algorithm uses the local estimations of the FB (Fire Behavior) algorithm, the speed of the wind and its velocity and the topography of the terrain. The proposed fuzzy logic system is show on Figure 5.

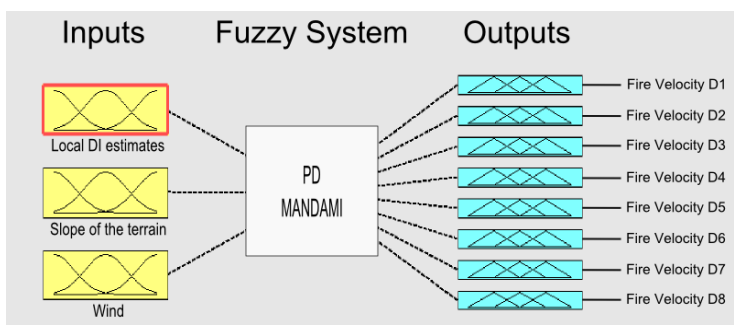

Figure 5: Representation of the PD Algorithm.

The fuzzy system uses two internal parameter calculated based on the local estimation of the direction of fire: the consensus direction of fire $(\mathrm{DFc})$ and the consensus velocity of fire $(\mathrm{VFc})$. Both are evaluated as the median of the local estimations of the system.

Table 8 summarizes the implementation rules. Due to the large number of rules that the system has, Table 8 only represents a summary of these. All others rules can be obtained along the same lines shown in this table.

Table 8: Rules of PD Algorithms.

\begin{tabular}{|c|c|c|c|}
\hline $\mathrm{DFc}$ & VIc & Slope & Outputs \\
\hline \multirow{8}{*}{$\begin{array}{c}\mathrm{DFc}= \\
\mathrm{D} 1\end{array}$} & \multirow{3}{*}{ Low } & D1=low & $\mathrm{D} 1, \mathrm{~V} 1=$ very low \\
\hline & & D1=medium & $\mathrm{D} 1, \mathrm{~V} 1=$ low \\
\hline & & D1=high & D1, V1 = medium \\
\hline & \multirow{3}{*}{ Medium } & $\mathrm{D} 1=$ low & $\mathrm{D} 1, \mathrm{~V} 1=$ low \\
\hline & & D1=medium & $\mathrm{D} 1, \mathrm{~V} 1=$ medium \\
\hline & & D1=high & D1, V1 = high \\
\hline & \multirow{2}{*}{ High } & D1=low & D1, V1 = high \\
\hline & & D1=high & $\mathrm{D} 1, \mathrm{~V} 1=$ very high \\
\hline \multirow{7}{*}{$\begin{array}{c}\mathrm{DFc}= \\
\mathrm{D} 4\end{array}$} & \multirow{7}{*}{ High } & D1=low & $\mathrm{D} 1=$ off \\
\hline & & D1 $=$ medium & $\mathrm{D} 1, \mathrm{~V} 1=$ low \\
\hline & & D1=high & D1, V1 = medium \\
\hline & & D3=low & $\mathrm{D} 3, \mathrm{~V} 3=$ low \\
\hline & & D3=medium & D3, V3 = medium \\
\hline & & \multirow{2}{*}{ D3=high } & D3, V3 = high \\
\hline & & & D4, V4= very high \\
\hline
\end{tabular}




\subsection{Infrastructure of ISFPWSN}

ISFPWSN is made up of two devices: anchor nodes and portable devices. A brief description about them is given below.

\subsubsection{Anchor Nodes}

Anchor nodes are made up of wireless sensors that form a wireless sensor network. These devices can communicate among each other using the appropriate routing protocols. The nodes execute all the algorithms of ISFPWSN. Every Anchor node obtains the next measures of sensor which include: temperature, wind direction, wind velocity, daily rain and humidity.

This information can be obtained from a small and cheaper weather station attached to each node, where the node can gather the information through a communication port, such as RS232-C or RS-485. It is interesting to provide power for these systems using renewable energy sources, such as solar panels or wind energy. Figure 6 shows an example that uses solar energy.

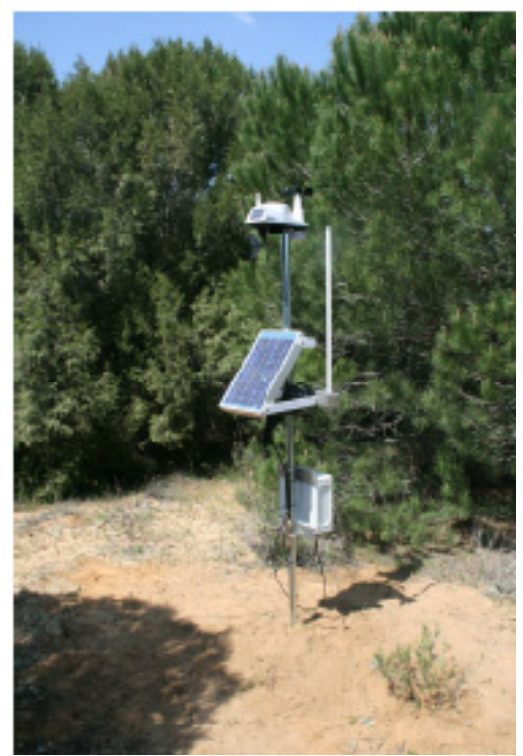

Figure 6: Example of an anchor node.

\subsubsection{Portable Devices}

The other devices used in the project are the measurement apparatus. They are a small and portable devices used by the fire-fighters to obtain the information of the environment. These devices can access all information on the state of the fire and can help in case of fire to provide information about its future evolution. This information is useful both to the extenuation and to determine secured ways for escape; increasing the safety of the workers.

\section{SYSTEM SIMULATOR}

In order to test the proposed algorithms a C++ adhoc simulator has been developed. Figure 7 shows the main windows of the simulator. This simulator has two classes of inputs: static inputs and dynamic inputs.

Static inputs represent characteristic of the terrain and the topology of the network. For the first study we have considered localization on the "Pinsapar" (see Figure 8) on the natural park of Grazalema, Cádiz, Spain. These variables cannot change on execution time. The topology of the terrain is summarized in Table 9.

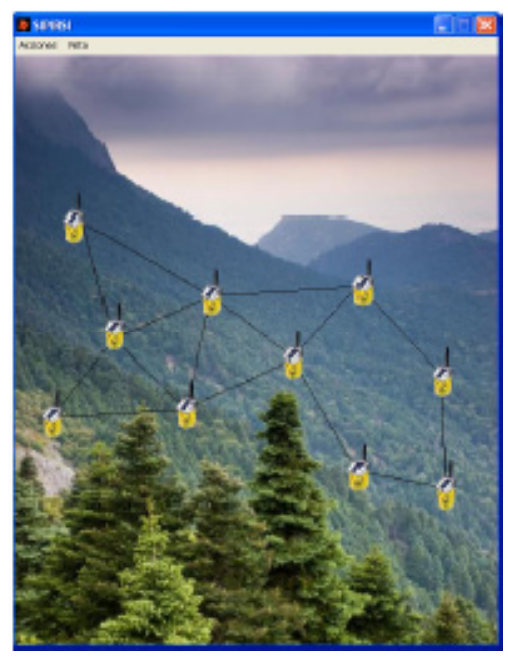

Figure 7: Main window of the simulator.

Table 9: Topology of the localization of the nodes.

\begin{tabular}{|c|c|c|}
\hline Node & Slope (\%) & Orientation $\left(\right.$ North $\left.^{\mathbf{}}\right)$ \\
\hline 1 & 38.6 & 220 \\
\hline 2 & 41.8 & 190 \\
\hline 3 & 37 & 182 \\
\hline 4 & 15 & 190 \\
\hline 5 & 21.8 & 235 \\
\hline 6 & 25 & 220 \\
\hline 7 & 27 & 170 \\
\hline 8 & 25 & 220 \\
\hline 9 & 33 & 235 \\
\hline 10 & 12 & 180 \\
\hline
\end{tabular}

Dynamic variables are the measures of the sensors of every node. The information can be change on the window of configuration of the 
sensors (see Figure 9). This window appears when you double click over a sensor of the main window.

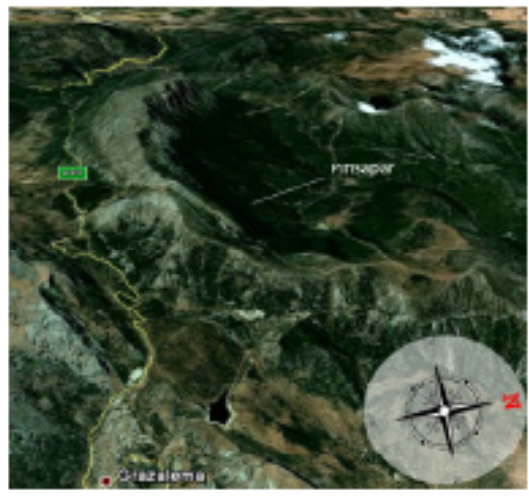

Figure 8: Localization considered for the simulations.

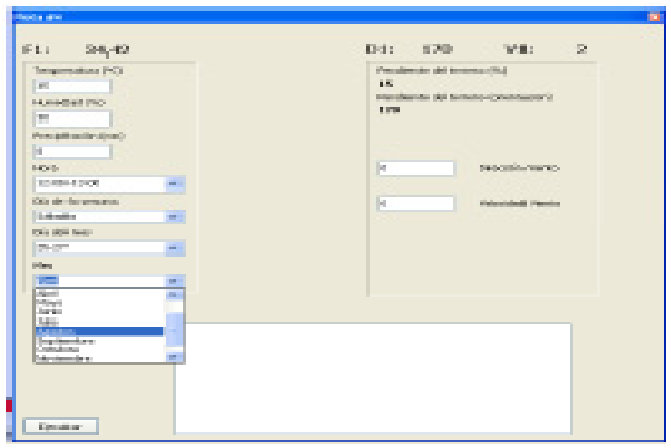

Figure 9: Window with the configuration of a node.

This simulator can show a graphic representation of the risk of fire (Figure 10) and the direction of fire (Figure 11).

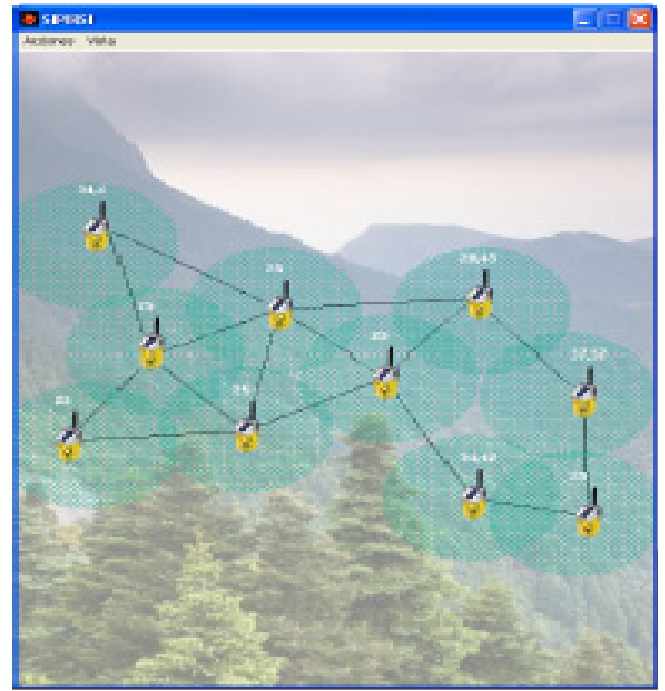

Figure 10: Window with the representation of the risk of fire.
All the partial and global results of the simulations can be obtained on a console window (see Figure 12).

With this simulator, ISFPWSN algorithm has been verified. In all of the simulated scenarios, ISFPWSN offers a correct response. Its response is similar to that obtained by BehavePlus simulator using the same inputs.

\section{CONCLUSIONS AND FUTURE WORK}

In this work, the ISFPWSN system is presented. It is a system that uses collaborative processing techniques over wireless sensor networks in order to cooperate with the wildfire fighting. This system can act in two ways: first, it can determinate the risk of fire and, in such a case, this network gives useful information to the fire fighters.

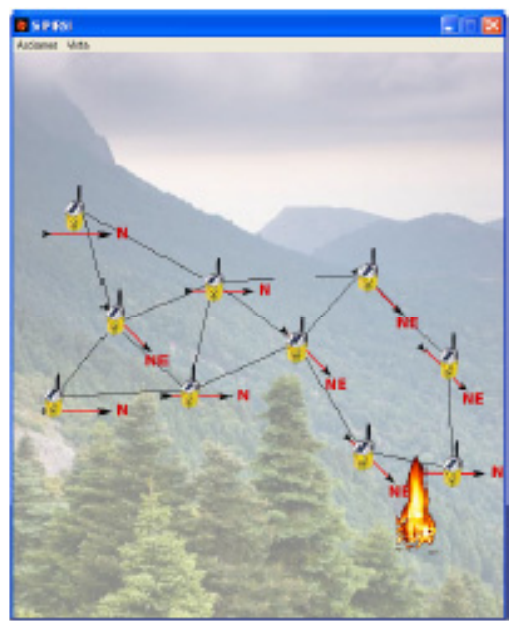

Figure 11: Window with the representation of the direction of a fire.

In case of fire, the system gives information about the location and direction of the fire fronts. This information can be used to study in effective manner the control of the fire and to design escape routes in order to enhance the safety of workers.

All of this information can be obtained by a firefighter with a portable device that can communicate with the designed WSN. One aim of wireless sensor networks is to be economical, permitting the development of the system in a huge area with a low cost. As future works, we plan to build a prototype of the system on a real environment in order to test the proposed algorithms. 


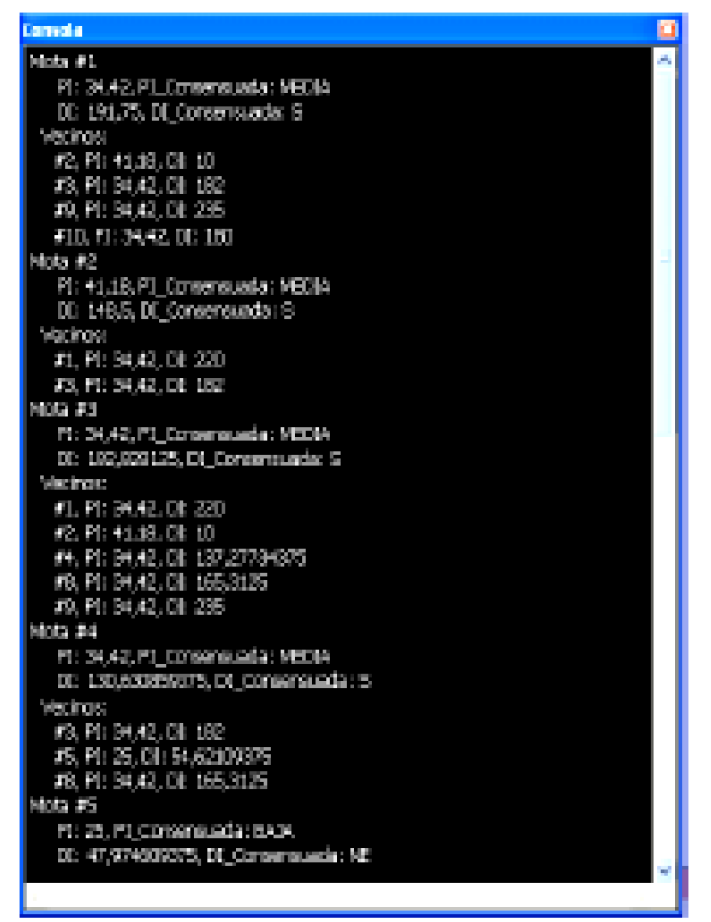

Figure 12: Window with console of result.

\section{ACKNOWLEDGEMENTS}

This work has been supported by the Consejería de Innovación, Ciencia y Empresa, Junta de Andalucía, Spain, through the excellence project ARTICA (reference number P07-TIC-02476).

\section{REFERENCES}

Akyildiz, I. F., Su, W., Sankarasubramaniam, Y., and Cayirci, E. (2002). Wireless sensor networks: A survey. Computer Networks, 38(4):393-422.

Antoine-Santoni, T., Santucci, J. F., de Gentili, E., Silvani, X. and Morandini, F. (2009). Performance of a protected wireless sensor network in a fire. Analysis of fire spread and data transmission. Sensors, 9(8):58785893.

Canadian Forest Fire Danger Rating System (CFFDRS). (1985). Equations and FORTRAN program for the Canadian Forest Fire Weather Index System.

Cherniak, A. and Zadorozhny, V. (2010). Towards adaptive sensor data management for distributed fire evacuation infrastructure. MDM'10. 11th International Conference on Mobile Data Managemet, pages 151-156.

García, E. M., Serna, M. A., Bermúdez, A., and Casado, A. (2008). Simulating a WSN-based wildfire fighting support system. ISPA'08. International Symposium on
Parallel and Distributed Processing with Application, pages 896-902.

Hefeeda, M., and Bagheri, M. (2007). Wireless sensor networks for early detection of forest fires. IEEE International Conference on Mobile Adhoc and Sensor Systems, pages 1-6.

Kosucu, B., Irgan, K., Kucuk, G., and Baydere, S. (2009). FireSenseTB: A wireless sensor networks testbed for forest fire detection. IWCMC'09, 6th International Conference on Wireless Communications and Mobile Computing: Connecting the World Wirelessly, pages 1173-1177.

Lei, Z., and Lu, J. (2010). Distributed coverage of forest fire border based on WSN. 2nd International Conference on Industrial and Information Systems, pages 341-344.

Li, W., Zhang, J., Zhang, J., Xia, Z., and Wang, G. (2009). The Monitoring of Host Computer for Forest Fire Detection System based on Wireless Sensor Network. WiCom'09, 5th International Conference on Wireless Communications, Networking and Mobile Computing, pages 1-4.

Liu, S., Tu, D., and Zhang, Y. (2009). Multiparameter fire detection based on wireless sensor network. ICIS'09. IEEE International Conference on Intelligent Systems, pages 203-206.

Marin-Perianu, M., and Havinga, P. (2007). D_FLER - A distributed fuzzy logic engine for rule based wireless sensor networks. LNCS 2007, vol. 4836; 86-101.

Obregón, P. D. P., Sondón, S., Sañu, S., Masson, F., Mandolesi, P. S., and Julián, P. M. (2009). System Based on Sensor Networks for Application in Forest Fire Prevention. EAMTA'09. Micro-Nanoelectronics, Technoloy and Applications, pages 61-65.

Ramachandran, C., Misra, S., and Obaidat M. S. (2008). A probabilistic zonal approach for swarm-inspired wildfire detection using sensor networks. International Journal of Comunication Systems, 21(10);1047-1073.

Rothermel, R. C. (1983). How to predict the spread and intensity of forest and range fires. US Department of Agriculture, Forest Service, General Technical Report, INT-143.

Yu, L., Wang, N., and Meng, X. (2005). Real-time Forest Fire Detection with Wireless Sensor Networks. International Conference on Wireless Communication, Networking and Mobile Computing, vol.2, pages 1214-1217.

Zhang, J., Li, W., Yin, Z., Liu, S., and Guo, X. (2009). Forest Fire Detection System based on Wireless Sensor Network. ICIEA'09, 4th IEEE Conference on Industrial Electronics and Applications, pages 520523. 